\title{
From Tools to Teammates: Conceptualizing Humans' Perception of Machines as Teammates with a Systematic Literature Review
}

\author{
Jennifer Rix \\ LMU Munich \\ rix@bwl.lmu.de
}

\begin{abstract}
The accelerating capabilities of systems brought about by advances in Artificial Intelligence challenge the traditional notion of systems as tools. Systems' increasingly agentic and collaborative character offers the potential for a new user-system interaction paradigm: Teaming replaces unidirectional system use. Yet, extant literature addresses the prerequisites for this new interaction paradigm inconsistently, often not even considering the foundations established in human teaming literature. To address this, this study utilizes a systematic literature review to conceptualize the drivers of the perception of systems as teammates instead of tools. Hereby, it integrates insights from the dispersed and interdisciplinary field of human-machine teaming with established human teaming principles. The creation of a team setting and a social entity, as well as specific configurations of the machine teammate's collaborative behaviors, are identified as main drivers of the formation of impactful human-machine teams.
\end{abstract}

\section{Introduction}

Traditionally, Information Systems (IS) research considers systems as tools that can be applied to achieve a certain outcome [1]. However, given the recent progress in Artificial Intelligence (AI), these systems may "soon be more than just tools to enhance team performance" $[2$, p. 1]. The accelerating capabilities of systems that enable the agentic and collaborative conduct of tasks require a change of perspective in the traditional user-system interaction paradigms. Contrary to the dystopic predictions of technology replacing humans, the notion of humans and machines teaming up to work collaboratively toward a shared goal has recently gained momentum [2,3]. Instead of humans taking the traditional role as an operator of the system, the user-system interaction becomes peer-like [4-7].

One form that this collaborative interaction could take is the formation of human-machine teams (HMTs). These HMTs already manifest in practice. The content creation domain is one prominent area in which HMTs already prevail [8]: Robo-journalists work alongside human journalists to create compelling content [9]. While human journalists leverage their wit and creativity to generate insights from data, the machine teammates utilize Natural Language Generation (NLG) to create drafts of narratives based on these insights [9]. Machine teammates are also leveraged in emergency response teams [2], as pilots [4], or also as teammates in card or video games $[10,11]$.

Research on human teams has revealed that team formation is a powerful tool for improving organizational outcomes [12]. Framing a group as a team fosters morale, commitment, and performance [13]. This also transfers to HMTs: When a system is framed as a teammate, the outcome of the interaction, as well as humans' acceptance of and affection for the system, improve [e.g. 7, 14-17]. Similarly, efficiency measures from human teaming literature were shown to be transferable to the HMT context. Team building measures, such as engaging in a collaborative, yet taskunrelated game, enhance a HMT's performance [7]. Consequently, designing and applying systems to foster their perception as teammates allows team formation's pervasive and powerful benefits to be realized also in the human-machine interaction context $[14,16]$.

Yet, research is inconclusive on the drivers of systems being perceived as teammates instead of tools. This lack of a consistent HMT conceptualization could be attributed to the dispersed research on HMTs: A variety of terms is used to refer to HMTs and insights are spread over multiple research disciplines. Further, "researchers are unaware of its specific requirements, and therefore underestimate the challenges of creating a team" [12, p. 489] which are introduced in human teaming literature. In the HMT context, teaming is often wrongly used as an analogue for any joint activity with a degree of interdependency, therefore disregarding the factors that drive a group's transformation into a team $[7,12]$. Further, since systems do not possess humans' innate capabilities, they also need to be configured to account for actions that would emerge naturally in 
human teaming, such as explaining choices or relationship building $[6,17,18]$. Thus, a consistent conceptualization of the teammate character of machines is required to leverage the benefits of HMTs:

Research Question: What drives humans' perception of systems as teammates instead of tools?

To answer this research question, we conducted a systematic literature review of extant HMT literature to integrate the fragmented research on HMTs. Further, extant research explicitly states that prevalent theories on human teaming need to be validated, translated, and potentially transferred to the HMT context [4, 18]. Accordingly, this review fills the gap of an integrated, holistic conceptualization of the drivers of machines' perception as teammates under the consideration of human teaming principles. This can serve as a basis for answering the multiple calls for research on humanmachine teaming in IS and Management [2, 13, 18, 19]. Moreover, when "machines evolve from tools to teammates, one thing is clear: accepting them will be more than a matter of simply adopting new technology" [20]. Understanding the basis of this new paradigm can thus also inspire the revision of established IS theories as desired by multiple IS scholars $[1,11,21]$.

\section{Theoretical foundations}

\subsection{Human-machine teams}

Human-machine teaming was first referred to as 'computers as teammates' in 1996 [15]. At time, research mostly focused on exploring IT artifacts as social actors and the capabilities of systems, respectively machines, were rather limited [12, 15]. These systems at a low level of automation (LOA) often lack collaborative capabilities and humans thus assume a supervisory role. Low LOA systems, therefore, can be attributed less of a teammate character [22, 23].

The "justification for redefining the team concept derives from the ability" [24, p. 1] of systems. AI capabilities allow machines to shift from being tools, with the human in a supervisory role, to becoming teammates [24]. Two recent developments have accelerated this shift. First, progress in AI allows for higher LOAs, whereby systems become increasingly agentic. While this enables systems to act independently, scholars agree that fully autonomous systems are not desirable as systems cannot handle unanticipated events well [3]. Yet, collaboration with humans can help systems overcome these innate problems. Second, the progress in AI also drives systems' collaborative capabilities. For example, given the progress in NLG [23], systems now have sophisticated communication capabilities.
These two developments in AI ultimately enable systems to become full-fledged teammates [1, 2, 21]. First, collaboration builds the basis for teamwork, and, accordingly, collaborative capabilities build the basis for systems to be perceived as teammates [25]. Second, increases in the LOA foster the perception of systems as teammates [24]. The hereby emerging prescriptive agency of systems allows them to take the roles of peers $[21,24,26]$. The latter implies that systems not only take over tasks but also take responsibility for all aspects of the task [5, 27]. This type of artifact is currently gaining momentum and is described as a turning point in IS research [21]. The question of what drives machines' perception as teammates instead of tools has therefore become particularly relevant given the recent generation of AI-enabled systems.

This newly emerging synergistic user-system interaction paradigm goes beyond the pure division of labor [28]. Instead, when a human perceives a system as a teammate, the human engages in teamwork processes that fundamentally differ from the unidirectional interactions in taskwork with a tool. The 'Big Five' core human teamwork processes that Salas et al. [29] mention also manifest in HMTs. For example, regarding the process of team leadership, it was shown that humans generally accept systems as leaders [18, 30, 31].

However, these beneficial teamwork processes only emerge when individuals perceive a teaming situation with a system. Humans recognizing a system as a teammate instead of a tool improves interaction outcomes as well as collaborative behaviors [e.g. 7, 14, 15, 32-34]. Further, teammate perceptions can enhance the acceptance of or even trust in a system [16, 17, 35]. Consequently, it is desirable to induce the perception of a system as a teammate, as this interaction paradigm leads to favorable outcomes compared to when a system is perceived as a tool.

Yet, the circumstances described to lead to this favorable perception of a system as a teammate are diverse and dispersed in HMT literature. There is a manifold of terms that imply teaming with a system, embracing more obvious terms like 'human-machine teams' [2], 'human-agent teams' [31], 'human-AI teams' [11], or 'human-autonomy teams' [4], but also 'hybrid intelligence' [36]. Forming a team with systems can also be easily confused with IT support for teams, such as by group decision support systems, or AI-based assistants as passive facilitators in workshops.

The hardship of creating a teaming perception with systems is demonstrated in a recent study with the aim to create a teaming perception with a system in a collaborative writing task [8]. Only half of this study's participants subsequently attributed teammate character to the system, despite its design science approach. The extensive research on human teaming has already 
established the drivers of team formation in a humanonly context. Nonetheless, many HMT studies neglect human teaming literature's requirements for team formation or only address fragments thereof $[7,12,31]$. Instead, teaming is often mistakenly used as an analogue for activities with a degree of interdependency [7, 12].

As the computers as social actors (CASA) paradigm postulates, humans tend to consider computers as social actors [15]. Accordingly, research often shows the transfer of human-to-human behavioral patterns and heuristics to the human-machine interaction context in line with the CASA paradigm or Social Response Theory [15, 37]. Based hereon, team formation as of human teaming literature should be considered as an initial theoretical lens for conceptualizing the factors driving humans to perceive systems as teammates instead of tools $[4,12,18]$.

\subsection{Theoretical framework}

The integration of communicative, coordinative, and cooperative actions leads to collaboration [25, 38]. Teams build on the foundation of collaboration, thus teaming can be considered a sub-form of collaboration [25]. Consequently, while collaboration acts as a prerequisite for team formation, other factors drive the perception of team formation. These team formation drivers change humans' perception of being part of a group to being part of a team [39], transforming taskwork into teamwork [25].

First, the formation of human teams requires the creation of a team setting, which emerges from the combination of frequently cited human team definitions. Four main factors were identified that drive the creation of a team setting: the presence of two or more individuals, shared goals, interdependency, and unique roles and functions [38-41]. Second, team formation requires the team's establishment and reinforcement as a social entity. Teams are described as such [39, 42], with team spirit and cohesion as integral components [43]. This entity is primarily formed by inducing team identity [44]. Team identity refers to teams being bounded, hence the members of the team having a clear understanding of who is part of the team and who is not [41]. Table 1 summarizes these team formation drivers.

However, it should be noted that human teaming formation drivers are not likely to suffice in the HMT context. Despite humans' tendency to perceive systems as social actors [37], they change their teaming behavior when a machine teammate is introduced. For example, team communication and coordination deteriorate when

\footnotetext{
${ }^{1}$ ["machines as teammates" OR "machine teammate" OR "synthetic teammate" OR "hybrid intelligence" OR "computers as teammates" OR "AI teammate" OR "human-machine team*" OR "human-
}

humans are told that their teammate is artificial $[5,24$, $34,45]$. This effect occurs regardless of the system's capabilities because it prevails even when a human operates the machine, i.e. in a Wizard-of-Oz (WOZ) scenario. Thus, the idiosyncrasies emerging in the HMT context should not be neglected, as the interaction with machine teammates might require additional, distinct team formation drivers. Accordingly, the integration of human team formation drivers with those explicated in HMT studies will enable a holistic conceptualization of the drivers of the perception of machines as teammates.

\section{Table 1. Human team formation drivers}

\begin{tabular}{|l|l|}
\hline Team setting & Description \\
\hline $\begin{array}{l}\text { Two or more } \\
\text { individuals }\end{array}$ & $\begin{array}{l}\text { A team should comprise at least two } \\
\text { team members. }\end{array}$ \\
\hline Shared goals & $\begin{array}{l}\text { Team members should work } \\
\text { toward a shared and valued goal. }\end{array}$ \\
\hline Interdependency & $\begin{array}{l}\text { Team members should contribute } \\
\text { reciprocally to the goal. }\end{array}$ \\
\hline $\begin{array}{l}\text { Unique roles and } \\
\text { functions }\end{array}$ & $\begin{array}{l}\text { Team members should be assigned } \\
\text { roles according to their strengths. }\end{array}$ \\
\hline Social entity & Description \\
\hline Team identity & $\begin{array}{l}\text { Team members should share an under- } \\
\text { standing of being part of the team. }\end{array}$ \\
\hline
\end{tabular}

\section{Methodology}

To ensure transparency and systematicity, this literature review follows the guidelines by Pare et al. [46]. The (1) underlying problem is the lack of a unified conceptualization of systems' teammate character. Given the different terms referring to HMTs, an 'understanding' review can help to integrate related concepts to conceptualize a phenomenon [47]. The (2) literature search comprised the databases EBSCOhost, AISeL, ScienceDirect, Taylor \& Francis, Web of Science, ACM DL, and Sage Journals. These were chosen as they list journals and conferences from the disciplines identified as most relevant in HMT research: IS, Human-Computer-Interaction, and Ergonomics. We accounted for quality by only including articles from peer-reviewed journals and established conferences (CHI, ECIS, HICSS, ICIS). Given the manifold terms for HMTs, we built the keyword set through an iterative process [48]. Using these keywords ${ }^{1}$ in a full-text search returned 931 distinct search results. The sample was then (3) screened for adherence to the following inclusion criteria: a) empirical research b) focused on user interaction with an IT artifact, whereby c) a teaming situation was explicated. We only considered

automation team*" OR "human-autonomy team*" OR "humancomputer team*" OR "human-agent team*" OR "human-AI team*"] 
systems in the roles of peers or experts, since facilitator roles are closely related to the system in a support role $[6,49]$. After screening, 24 publications remained. An additional forward and backward search yielded seven further publications. Consequently, the sample includes 31 publications. Since we only included peer-reviewed outlets, the (4) quality assessment requirement became obsolete. In terms of (5) analysis, we first coded the articles deductively along the human teaming principles listed in Table 1. We then used an inductive approach to identify and categorize distinctive HMT context features. Two researchers coded the articles to ensure inter-coder reliability.

\section{Results}

Of the 31 empirical studies in the sample, twothirds follow an experimental approach. The sample also includes taxonomy, design science, and interview studies. Intelligent agents and conversational agents form the foundation of all of these studies. Hereby, two distinct types of inquiry into HMT formation emerge. While some studies only address team formation indirectly in their research setting [e.g. 34], other studies use an explicit, explorative, and direct approach to investigate perceptions of systems as teammates in specific contexts, such as gaming [e.g. 11]. Two-thirds of the studies provide explicit definitions of HMTs, with only about half making references to human teaming.

Table 2 illustrates the deductive analysis of extant HMT literature in conjunction with human team formation drivers (see Table 1). The subsequent sections explain the manifestations of the team formation drivers as of human teaming in the HMT context and introduce HMT-specific drivers emerging from the HMT studies. Further, due to the idiosyncrasies of the HMT context, another dimension of team formation drivers emerged from the inductive analysis: collaboration behavior.

\subsection{Team setting}

The creation of a team setting drives humans' team formation perception. First, teams need to comprise more than two individuals. Dyadic studies are rather scarce in a HMT context [e.g. 10]. Humans also tend to favor forming three- rather than two-member HMTs [31]. The team composition also becomes relevant in a HMT context as two distinct forms emerge: multi-agent teams (i.e. only one human, but multiple synthetic agents) and multi-human teams (i.e. more than one human). Multi-agent teams demonstrate less team cognition [45]. Hence, including more than one human in a team can improve teaming perception, resulting in superior communication and coordination [45]. Given the study's WOZ setting, these insights are even detached from the machine teammates' behavior [45].

Second, almost all HMT publications also describe a shared and valued goal. However, an overall shared goal (e.g. resource optimization) does not rule out conflicting local goals (e.g. loss of own resources) [54]. HMTs' shared goals embrace optimizing resource allocation [54], drawing a picture of a prespecified object [35], or defeating an opponent in a gaming setting [33]. Yet, in hybrid intelligence, goals may be shared, but may also be adversarial (e.g. for opponents in a game) [36]. One study also considers non-goal-oriented teammates, with value derived from the interaction itself [49]. Nevertheless, as almost all studies in the sample consider these two determinants, they could be considered minimal prerequisites for HMT formation.

The majority of HMT publications also addresses interdependency as a driver of teammate perception. Analogously to the definition of human teaming [29], HMTs' interdependency may manifest itself in reciprocal interdependence when team members contribute to the goal in a modular manner [8, 33]. This reciprocity also induces bi-directionality of exchange, which was also described as a significant driver for recognizing machines as teammates [53]. In this respect, both, humans and systems exchange information as well as actions with the environment, but at the same time also involve in information and task exchange with each other $[17,52]$. Three further forms of interdependency emerge in HMTs. First, one team member's outcome may influence the entire team's outcome significantly, such as a shared monetary reward $[14,15]$. Second, the human team may be dependent on the machine with

Table 2. Adherence of HMT studies to human team formation drivers

\begin{tabular}{|c|c|c|c|c|c|c|c|c|c|c|c|c|c|c|c|c|c|c|c|c|c|c|c|c|c|c|c|c|c|c|c|}
\hline & & & & & & & & & & & & & & & & & & & & & & & & & & & & & & & \\
\hline Reference & $\Xi$ & $\sqrt{n}$ & $\sqrt{6}$ & $\Sigma$ & $\infty$ & 흘 & $\Xi$ & $\Xi$ & $\underline{\Omega}$ & $\underset{\sigma}{\Xi}$ & $\Xi$ & $\stackrel{\infty}{=}$ & $\underline{\mathbf{c}}$ & $\underset{\mathrm{d}}{\mathbf{\Xi}}$ & ¿ & 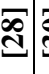 & & $\bar{\Xi}$ & ָิ & $\tilde{\omega}$ & $\vec{\Xi}$ & $\begin{array}{l}\bar{n} \\
\tilde{\theta}\end{array}$ & (ా) & & $\stackrel{9}{ \pm}$ & $\overline{8}$ & $\bar{\sigma}$ & $\begin{array}{l}\bar{N} \\
\boldsymbol{n} \\
\end{array}$ & ह̋ & 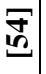 & \\
\hline \multicolumn{32}{|l|}{ Team setting } \\
\hline pre indi & $\mathrm{x}$ & $\mathrm{x}$ & $\mathrm{x}$ & $\mathrm{x}$ & $\mathrm{x}$ & $\mathrm{x}$ & $\mathrm{x}$ & \begin{tabular}{|l|}
$x$ \\
\end{tabular} & $\mathrm{x}$ & $\mathrm{x}$ & $\mathrm{x}$ & \begin{tabular}{l|l}
$x$ & 2 \\
\end{tabular} & $x$ & $\mathrm{x}$ & \begin{tabular}{l|l}
$x$ & $x$ \\
\end{tabular} & $\mathrm{x}$ & $x$ & $\mathrm{x}$ & $\mathrm{x}$ & $\mathrm{x}$ & $\mathrm{x}$ & $\mathrm{x}$ & $\mathrm{x}$ & $\mathrm{x}$ & $x$ & $x$ & \begin{tabular}{l|l}
$X$ & $x$ \\
\end{tabular} & $\mathrm{x}$ & $\mathrm{x}$ & $\mathrm{x}$ & $\mathrm{x}$ \\
\hline Shared goals & $x$ & $\mathrm{x}$ & $\mathrm{x}$ & $\mathrm{x}$ & $\mathrm{x}$ & $\mathrm{x}$ & $\mathrm{x}$ & $\mathrm{x}$ & $\mathrm{x}$ & $\mathrm{x}$ & $\mathrm{x}$ & \begin{tabular}{l|l}
$\mathrm{X}$ & $\mathrm{x}$ \\
\end{tabular} & $\mathrm{x}$ & $\mathrm{x}$ & \begin{tabular}{l|l}
$\mathrm{X}$ & $\mathrm{x}$ \\
\end{tabular} & $\mathrm{x}$ & $x$ & $\mathrm{x}$ & $x$ & $\mathrm{X}$ & $\mathrm{x}$ & $x$ & & $\mathrm{X}$ & & $x$ & $\mathrm{x}$ & $\mathrm{x}$ & $\mathrm{x}$ & $\mathrm{x}$ & $\mathrm{x}$ \\
\hline Interdependency & $\mathrm{x}$ & $\mathrm{x}$ & $\mathrm{X}$ & $\mathrm{x}$ & $\mathrm{x}$ & $\mathrm{x}$ & $\mathrm{x}$ & $\mathrm{X}$ & $\mathrm{x}$ & $\mathrm{x}$ & $\mathrm{x}$ & \begin{tabular}{l|l}
$\mathrm{X}$ & $\mathrm{x}$ \\
\end{tabular} & $\mathrm{x}$ & $\mathrm{x}$ & $\mathrm{X}$ & & $x$ & $\mathrm{x}$ & & $\mathrm{X}$ & $\mathrm{x}$ & $\mathrm{x}$ & $\mathrm{x}$ & $\mathrm{x}$ & & $x$ & $\mathrm{X}$ & $\mathrm{X}$ & $\mathrm{X}$ & $\mathrm{x}$ & $\mathrm{x}$ \\
\hline Unique roles and & $\mathrm{x}$ & $\mathrm{x}$ & $\mathrm{x}$ & & & & $\mathrm{x}$ & & & & $\mathrm{x}$ & \begin{tabular}{l|l}
$x$ & $x$
\end{tabular} & $x$ & \begin{tabular}{l|l}
$x$ \\
\end{tabular} & \begin{tabular}{l|l}
$x$ & $x$
\end{tabular} & $\mathrm{x}$ & $\mathrm{x}$ & & & & $\mathrm{x}$ & $\mathrm{x}$ & $\mathrm{x}$ & & & $\mathrm{x}$ & $\mathrm{x}$ & $\mathrm{x}$ & & & \\
\hline \multicolumn{32}{|l|}{ Social entity } \\
\hline Team identity & & & & $\mathrm{x}$ & $\mathrm{x}$ & & $\mathrm{x}$ & $\mathrm{x}$ & $\mathrm{x}$ & & $\mathrm{x}$ & & & & & & & & & & & & & & & & & & & & \\
\hline
\end{tabular}


regard to its performance. This dependence occurs when "the synthetic teammate cannot be set aside and the team expected to perform the task well" [5, p. 4]. This dependence is magnified in a synergistic teaming scenario based on the teammates' unique or complementary skills [45], but also when humans' cognitive resources are freed up [55]. Lastly, a machine may also take a transactional leadership role [30, 51]. Overall, creating a form of interdependence can prevent machine teammates from being siloed, thus fostering humans' perception of them as teammates [45].

Unique roles and functions in order to create a team setting find less resonance in a HMT context. In human teaming, roles are assigned on the basis of the team members' respective capabilities [36]. Some HMT studies assign machine teammates to complementary roles, where machines can, for example, assume a unique role as an expert [49] or a leader [30, 51]. Yet, machine teammates are often investigated in the same roles as their human teammates. Hereby, the machine teammates take a peer role [49], and have abilities that match those of their human team members [10,31]. This tendency toward peer roles may either result from the machines being seen as work-relief $[32,55]$ or abstract experimental settings [14, 15]. Accordingly, in a HMT setting, it seems to be of greater relevance for the perception of team formation that the machine teammate takes a role at all, which, however, does not have to be unique [50]. The latter implies that both parties are engaged in co-creating the final outcome in equal parts $[5,6,35]$. For example, in a drawing task, the human may take the role of starting the sketch and suggesting colors, while the machine completes the sketch and colors it in [35].

\section{Table 3. HMT-specific team setting drivers}

\begin{tabular}{|l|l|}
\hline Driver & Description \\
\hline $\begin{array}{l}\text { Team } \\
\text { composition }\end{array}$ & $\begin{array}{l}\text { The team should comprise at least two } \\
\text { humans. }\end{array}$ \\
\hline Co-creation & $\begin{array}{l}\text { Team members should take roles that } \\
\text { contribute to attaining the overall goal. }\end{array}$ \\
\hline
\end{tabular}

\subsection{Social entity}

Second, establishing the team as a social entity also drives humans' team formation perception. Creating a team identity can foster this social entity. Yet, creating a team identity is less prevalent in HMT research [6, 14]. The manifestation of team identity implies humans' explicit attribution of team membership to, and thus anthropomorphization of, systems $[15,17]$. Hereby, the team identity may lead to the feeling of social presence, such as when participants felt "as if they were with someone" [35, p. 9]. Team identity can be manipulated by team labels, such as team 'red' or 'blue' $[14,15]$, or by the machine introducing itself as a teammate [8]. Yet, team identity manipulations by team labels were only effective as long as another team was present $[14,15]$.

Since machines do not possess human team members' inherent characteristics and behaviors, HMT research also explicates the need to foster the system's anthropomorphization in order to build rapport and nudge humans to perceive the machine as a viable social actor [18, 49]. First, all qualitative inquiries in the sample state humanness to be critical for machines' recognition as teammates $[6,8,11]$. This may be fostered by a name for the machine teammate [51], its embodiment in the form of an avatar [51], graphical typing indicators $[8,28]$, the exhibition of emotions [49], implicature in communication [10], or a degree of unpredictability in conversation and actions [11, 35]. Machines' transparency regarding their social intent (i.e. benevolence) [17] and procedural justice [30] further enhance their perceived humanness in a HMT context. This not only promotes the perceptions of machines as teammates, but also enhances team trust, performance, and experience $[11,17,35]$.

In addition to humanness, relationship-building behavior would emerge naturally in human teams but needs to be explicitly configured in machine teammates. Relationship-building behavior may manifest in machine teammates engaging in off-task conversation [6] or establishing a shared understanding of a task [50]. Also, informal team building, such as jointly playing a collaborative game before interacting as a team, has proven effective for enhancing HMT performance [7]. Consequently, these two additional drivers foster the HMT's manifestation as a social entity, which, in turn, enhances humans' willingness to hand tasks to and engage in teamwork with machines [32].

\section{Table 4. HMT-specific social entity drivers}

\begin{tabular}{|l|l|}
\hline Driver & Description \\
\hline Humanness & $\begin{array}{l}\text { Machine teammates should exhibit a } \\
\text { certain degree of humanness. }\end{array}$ \\
\hline $\begin{array}{l}\text { Relationship } \\
\text { building }\end{array}$ & $\begin{array}{l}\text { Machine teammates should exhibit } \\
\text { relationship-oriented behavior. }\end{array}$ \\
\hline
\end{tabular}

\subsection{Collaboration behavior}

Machines' collaboration ability can be seen as a prerequisite for their perception as teammates alongside their ability to act independently. These collaborative behaviors can be configured in different ways. Some of these configurations were even suggested to act as drivers of the perception of a machine as a teammate. Thus, while collaborative capabilities are generally an enabler of teaming, specific collaborative behaviors can even act as drivers. Since humans already exhibit these behaviors innately, the configuration of collaboration 
behavior emerges as an additional dimension of team formation drivers in the HMT context.

First, machine teammates should exhibit proactive behavior, hence no team member should exclusively lead the interaction [6]. Proactivity also entails machine teammates' ability to take the initiative and request actions from a human [4, 6, 11, 24]. In HMTs, proactivity may manifest in pushing as well as pulling information of all involved parties [6, 34, 52, 54]. The machine may for example introduce itself proactively or ask the human to provide support [8, 28]. Proactivity may also be dependent on the machine's respective role, as machine teammates in expert roles are often designed to take a passive role [49]. This proactivity provides the basis for attributing (prescriptive) agency to the machine, driving its perception as a teammate [21].

Second, designing the system for interactions in iterative behavior is described to enhance teaming perceptions [8]. This requires humans and machines to take turns, therefore creating multiple points of contact in respect of attaining the goal $[4,8,26,35]$. Hereby, the human should also recognize that the machine counterpart is working, which may be operationalized with a time delay and graphical representation of the machine teammate working [8].

Third, humans have expressed their preference for NLG-based communication in HMTs [11, 28]. Yet, although text-based communication dominates the sample, experimental evidence suggests that voicebased communication has more positive effects on the perception of teaming and team performance than interface- or text-based communication [23]. Further, regarding communication style, naturalness [6] and a high level of detail [35], as well as implicit communication $[10,45]$ are desired in HMTs.

Fourth, machine teammates should withstand dynamic environments, hence exhibit responsive behavior. This responsiveness manifests itself in machine teammates' adaptiveness [24, 26] and learning capabilities [36]. Adaptiveness allows for the continuous revision of the shared goal and the ability to satisfy unexpectedly emerging demands of human teammates [53]. The importance of adaptiveness is further underlined by its recognition as a 'Big Five' human teamwork process [29]. Learning capabilities, which are especially highlighted in the notion of hybrid intelligence, allow a HMT to co-evolve and improve over time by continuously learning from each other, resulting in symbiotic co-evolution $[36,55]$.

Fifth, explanation provision is described as important for recognizing machines as teammates. In HMTs, it is essential to manage expectations $[8,11]$ and establish bi-directional transparency [50]. Machine teammates are described as inherently unpredictable, even in a WOZ scenario [45]. In a survey study, more than $80 \%$ of the respondents believed that explanations of the machine teammate's actions would result in superior team performance, which in turn may drive humans' willingness to recognize the machine as a teammate [11]. To address this, the machine could explain its functionality when introducing itself to the human [8] or provide detailed instructions for the teamwork [35]. Besides, explanations are crucial for backup behavior - also a 'Big Five' teamwork process and can foster shared mental models and trust [17, 50].

Lastly, competent behavior of the machine is named as a driver of teaming perception. This can again be attributed to an idiosyncrasy of the HMT context: While humans innately possess general intelligence, systems do not. Extant research shows that humans do not expect machines to be perfect or possess general intelligence $[8,11]$. Perceiving machines as competent in an area of expertise [8], or even slightly better at a specific task, is sufficient for benefitting its recognition as a teammate [11]. A machine may also fulfill the same tasks as humans do, and hence have similar skills [8, 33, 54]. Yet, qualitative research suggests that superior or complementary skills may foster machines' perception as teammates $[6,11]$. Hereby, the machine should be innovative and detail-oriented [18], but also exhibit selfdepreciation and acknowledge the need to learn [8].

\section{Table 5. HMT-specific collaboration behavior drivers}

\begin{tabular}{|l|l|}
\hline Driver & Description \\
\hline $\begin{array}{l}\text { Proactive } \\
\text { behavior }\end{array}$ & $\begin{array}{l}\text { Machine teammates should take } \\
\text { initiative independently. }\end{array}$ \\
\hline $\begin{array}{l}\text { Iterative } \\
\text { behavior }\end{array}$ & $\begin{array}{l}\text { Machine teammates should be able to } \\
\text { structure their work process to allow } \\
\text { for turn-taking when collaborating. }\end{array}$ \\
\hline $\begin{array}{l}\text { Voice-based } \\
\text { communication }\end{array}$ & $\begin{array}{l}\text { Machine teammates should have } \\
\text { voice-based NLG capabilities. }\end{array}$ \\
\hline $\begin{array}{l}\text { Responsive } \\
\text { behavior }\end{array}$ & $\begin{array}{l}\text { Machine teammates should adapt to } \\
\text { and learn from environmental changes. }\end{array}$ \\
\hline $\begin{array}{l}\text { Explanation } \\
\text { provision }\end{array}$ & $\begin{array}{l}\text { Machine teammates should be able to } \\
\text { articulate their reasons for their actions. }\end{array}$ \\
\hline $\begin{array}{l}\text { Competent } \\
\text { behavior }\end{array}$ & $\begin{array}{l}\text { Machine teammates should possess } \\
\text { superior skills in certain tasks. }\end{array}$ \\
\hline
\end{tabular}

\section{Discussion}

Subsequently, we critically discuss the relevance of the individual factors mentioned in section 4. This serves as the basis for the derivation of propositions conceptualizing the drivers that lead humans to perceive systems as teammates instead of tools [48].

Concerning the creation of a team setting, studies could operationalize interdependency in achieving a shared goal to elicit teammate affiliations in HMTs [1416]. Moreover, the team composition should ideally 
involve at least two human team members [45]. However, team formation perceptions were elicited even when humans and machines took the same role, hence no unique roles and functions prevailed [8]. Instead, co-creation seems to be more relevant. While a machine teammate's role does not have to be unique, the machine teammate needs to have a dedicated role that contributes to the outcome. For example, a virtual assistant may make proactive suggestions and execute recommendations once a human has approved them, yet does not qualify as a teammate due to its lack of a dedicated role [6]. In contrast, in a collaborative writing task, the machine may assume the role of a contributor of individual sentences. In this case, the machine and the human teammates take the same role, yet the machine actively participates in the joint, iterative co-creation of a story [8]. This ultimately sets machine teammates apart from merely supporting humans in task conduct. Co-creation thus replaces unique roles and functions in the HMT context. Proposition 1 summarizes the factors fostering a team setting for HMTs.

Proposition 1: To create a team setting, HMTs should comprise at least two humans working with a machine teammate in interdependent roles designed for co-creation toward a shared goal.

Concerning the formation of a social entity as in human teaming, the effect of creating a team identity for HMTs can only be operationalized in the presence of other teams and in abstract research settings [14, 15]. Although we may generally perceive machines as social actors as suggested by the CASA paradigm, the machines need to be configured in ways that will enhance their recognition as a viable part of a social entity $[6,8,11]$. The machines' anthropomorphization is pivotal in this process. The integration of human-like characteristics, for example through an embodied avatar [8] or implicit communication [10], have proven effective in fostering teammate affiliation to machines. Relationship-oriented action patterns, such as inducing team building [7] or task-unrelated communication [11], have likewise proven effective in this regard. These contentions are also in line with the claim that Social Response Theory becomes more important as a kernel theory [6, 37]. Human-like and relationship-building configurations reinforce the perception of machine teammates as social actors, which, in turn, may make it easier for humans to identify with their machine teammate. Consequently, the effectiveness of team identity manipulations may recuperate. Proposition 2 summarizes the drivers of social entity formation.

Proposition 2: To establish the team as a social entity, team identity cues should be paired with machine teammates exhibiting humanness and involving in relationship building.
Collaborative interaction is suggested as the primary enabler of team formation in human teams. Although collaborative capabilities are regarded as enablers for machines to act as teammates, not all three collaborative activities - communication, coordination, and cooperation - are attributed similar attention in the HMT context. Only a few HMT studies in the sample explicitly address cooperation [e.g. 53, 54]. A potential reason for the lack of explication of cooperation is that machines do not possess the innate irrationality and subjectivity that might prevent humans from cooperating. Instead, communication and coordination are seen as the critical activities in HMTs [45].

While machines' collaborative capabilities allow them to act as teammates, specific collaborative behaviors drive humans' perception of team formation with machines. Humans have the innate ability to exhibit various collaborative behaviors, such as explaining their behavior or proactively engaging in teamwork, but machines need to be configured to take these collaborative behaviors into account. Thus, a third driver dimension emerges in the HMT context.

Machines exhibiting proactive and iterative collaborative behaviors, resulting in self-initiated turn taking with multiple points of contact between the teammates, drive humans' perceptions of machines as teammates [6, 8]. Voice-based rather than text-based communicative behaviors reinforce these perceptions [23]. In addition, responsive behavior, as reflected in machines that adapt their collaborative behavior to environmental cues and learn from experience, further fosters teammate perceptions [24, 36]. The machine teammate should explain these changes in collaboration behavior to counter the unpredictability attributed to it [11]. Lastly, humans should regard their machine teammate as exhibiting competent behavior [6]. Proposition 3 summarizes these configurations.

Proposition 3: To induce collaborative behavior that fosters teammate perceptions, machine teammates should exhibit proactive, iterative, responsive as well as competent behavior while providing explanations and allowing for verbal communication.

Figure 1 summarizes the drivers of HMT formation perceptions. This figure displays the human teaming drivers applicable in the HMT context as well as the specific drivers emerging in this context. It becomes apparent that the human teaming drivers are extended by a larger number of HMT-specific drivers. This again underlines the need to clearly conceptualize HMT formation drivers due to the idiosyncrasies and complexity of the HMT context. Hereby, the prevalence of all three dimensions of team formation drivers is required for perceiving machines as teammates instead of tools. For example, systems may exhibit the collaborative behaviors of machine teammates, but may 
still be regarded as tools if a team setting and a social entity are not induced [11]. It is therefore the triad of determinants that sets human-machine teaming apart as a subform of human-machine collaboration. This way, also concepts such as human-machine symbiosis or human-in-the-loop can be clearly delineated from HMTs. Nevertheless, while this conceptualization provides a holistic picture, it remains for further investigation which factors act as the strongest drivers.

\section{Contribution}

In this paper, we integrated distinct HMT concepts with human team formation prerequisites to understand this diverse and interdisciplinary research field [47]. The derived insights delineate the factors that drive systems' perceptions as teammates instead of as tools. This is especially important given the conflicting HMT conceptualizations. Although there have been inquiries into machines' conceptualization as teammates, they either focus on a specific system type [27], context [6], or research discipline [22]. Further, the human teaming drivers are often neglected in the HMT context, although they are well-researched [7, 12]. To the best of our knowledge, this study is the first to provide a holistic conceptualization of the drivers that make machines teammates under consideration of human teaming.

Regarding the theoretical contributions, this study first creates a unified, coherent basis for further research on the HMT notion. The underlying study allows for differentiating a teaming situation with a system from the traditional use of a system as a tool. This is especially important given the multiple calls for qualitative and practice-oriented research on team formation with machines [2, 13, 18, 19]. Further, this conceptualization helps to identify studies that arbitrarily use the term 'teaming' as a buzzword [7, 12]. For example, Poser and Bittner [27] contend that, in their review, no conversational agent could fully engage as a teammate and in teamwork. This conceptualization, therefore, provides an informed basis for future research in the HMT field and allows extant literature to be assessed regarding the prevalence of teaming situations.

Second, the conceptualization of machines as teammates can help the theorizing on the 'new generation of use' that currently prevails in IS [1, 21]. Teaming with systems is in contrast with the traditional notion of systems as tools, whereby teaming is a subform of the newly emerging bilateral user-system interaction paradigm that progress in AI has enabled [1]. In unilateral interactions, a human provides a request and a machine delivers a result, while in bilateral interactions, both parties push and pull information and exchange their work products [1, 17]. The conceptualization of machines as teammates also accounts for the increasingly (prescriptive) agency of machines [2, 21]. Consequently, well-researched technology use concepts need to be adjusted to account for this new form of user-system interaction. For example, shared mental models need to be "expanded to incorporate teamwork" [50, p. 31]. Similarly, distinct drivers of trust emerge in HMTs: integrity and benevolence [4]. Thus, this study can inspire the revision of established IS theories and aid the theorizing of novel paradigms for user-system interaction in IS.

Third, the unveiled similarities between HMTs and human teams make the deep-pocketed research on human teaming eligible for transfer in the IS context. Teamwork processes are "unfamiliar territory" [17, p. 175] in technology-focused literature. Since accepting machine teammates "will be more than a matter of simply adopting new technology" [20], the humanteaming literature can be used to identify, describe, and leverage these newly emergent behaviors with this new generation of AI-enabled systems. Research already provides evidence for the effectiveness of transferring measures for team effectiveness from human teaming to HMTs. Even informal team building - a concept intuitively only applicable in human teaming - has proven effective in a HMT context [7].

Regarding the practical contributions, this study guides the system design of machine teammates by

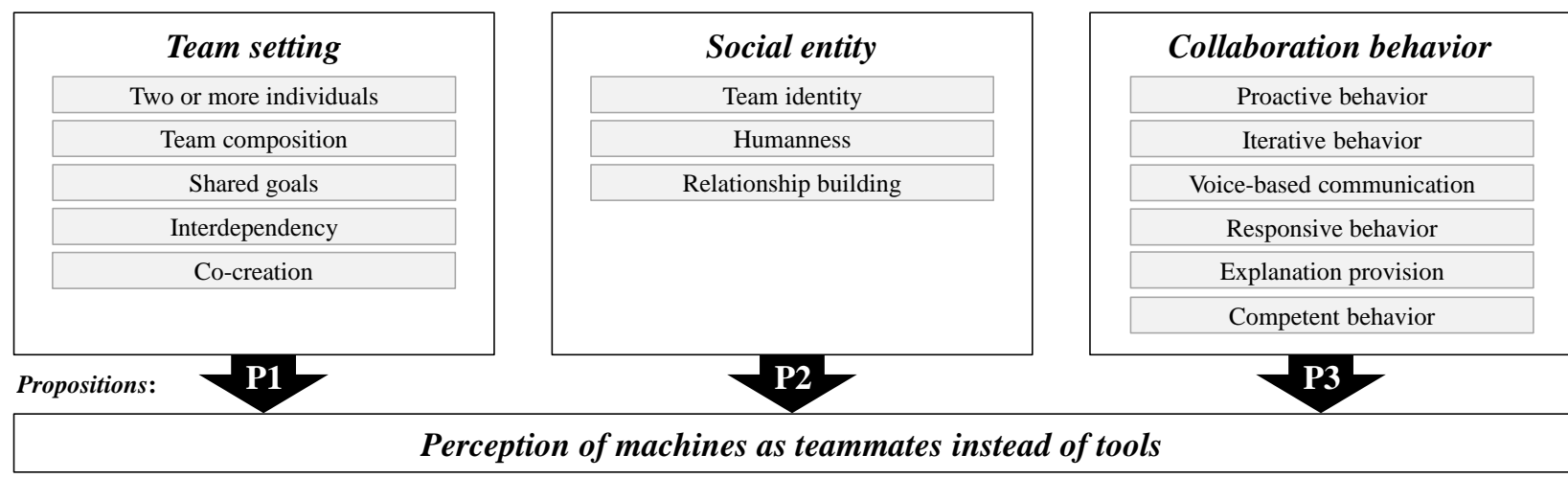

Figure 1. Drivers of the perception of machines as teammates instead of tools 
identifying the drivers of teammate perceptions [11]. Systems inducing these perceptions can, for example, improve interaction outcomes [7]. Further, this study allows identifying HMT situations in organizations. Since teaming may have distinct implications compared to tool usage, it enables more effective management and anticipation of potential issues [2].

\section{Limitations and future research}

Despite a high degree of systematicity, this study is not without limitations. First, while it provides insights into the drivers of HMT formation based on extant literature, it does not empirically assess their respective importance and interactions. Future research should empirically validate the identified drivers. Second, we integrated insights from various contexts. For example, in an organizational context, it may be even harder for systems to convince humans to think of them as teammates, as employees may feel that these systems threaten their identity [3]. Further, a large share of HMT studies is set in highly controlled or military contexts $[17,45]$. The generalizability of these findings thus needs to be validated. Third, mandated by space restrictions, future research could extend the breadth of the keyword set. For example, this research's scope does not take the complexity that physical presence induces into account [22]. Yet, insights from human-robot teaming and autonomous vehicles may prove valuable. Fourth, an overview of the relationships investigated in a HMT context could extend the depth of the analysis. This would allow a clearer illustration of the differences between teaming and use since it will reveal research constructs specific to HMTs. Also, HMT-specific behaviors can be addressed, such as machine teammates being treated less fairly [31].

\section{References}

[1] S. Schuetz, and V. Venkatesh, "The rise of human machines: How cognitive computing systems challenge assumptions of user-system interaction", Journal of the Association for Information Systems, 21, 2020, pp. 460-482. [2] I. Seeber, E. Bittner, R.O. Briggs, T. De Vreede, G.-J. De Vreede, A. Elkins, R. Maier, A.B. Merz, et al., "Machines as teammates: A research agenda on AI in team collaboration", Information \& Management, 57(2), 2020, pp. 1-22.

[3] L. Willcocks, "Robo-Apocalypse cancelled? Reframing the automation and future of work debate", Journal of Information Technology, 35(4), 2020, pp. 286-302.

[4]* N.J. McNeese, M. Demir, E.K. Chiou, and N.J. Cooke, "Trust and team performance in human-autonomy teaming", International Journal of Electronic Commerce, 25(1), 2021.

[5]* N.J. McNeese, M. Demir, N.J. Cooke, and C. Myers, "Teaming with a synthetic teammate: Insights into humanautonomy teaming", Human Factors, 60, 2018, pp. 262-273.
[6]* E. Elshan, and P. Ebel, "Let's team up: Designing conversational agents as teammates", Proceedings of the $41^{\text {st }}$ International Conference on Information Systems, 2020.

[7]* J.C. Walliser, E.J. de Visser, E. Wiese, and T.H. Shaw, "Team structure and team building improve human-machine teaming with autonomous agents", Journal of Cognitive Engineering and Decision Making, 13(4), 2019, pp. 258-278. [8]* C. Wiethof, N. Tavanapour, and E. Bittner, "Implementing an intelligent collaborative agent as teammate in collaborative writing: Toward a synergy of humans and AI", Proceedings of the $54^{\text {th }}$ Hawaii International Conference on System Sciences, 2021.

[9] K.N. Dörr, "Mapping the field of algorithmic journalism", Digital Journalism, 4(6), 2016, pp. 700-722.

[10]* C. Liang, J. Proft, E. Andersen, and R.A. Knepper, "Implicit communication of actionable information in humanAI teams", Proceedings of the $37^{\text {th }}$ CHI Conference on Human Factors in Computing Systems, 2019.

[11]* R. Zhang, N.J. McNeese, G. Freeman, and G. Musick, ""An ideal human": Expectations of AI teammates in humanAI teaming", Proceedings of the ACM on Human-Computer Interaction, 4(CSCW3), 2021, pp. 1-25.

[12] V. Groom, and C. Nass, "Can robots be teammates?: Benchmarks in human-robot teams", Interaction Studies, 8(3), 2007, pp. 483-500.

[13] L. Larson, and L.A. DeChurch, "Leading teams in the digital age: Four perspectives on technology and what they mean for leading teams", The Leadership Quarterly, 31(1), 2020, pp. 1-18.

[14]* D. Johnson, and J. Gardner, "The media equation and team formation: Further evidence for experience as a moderator", International Journal of Human-Computer Studies, 65(2), 2007, pp. 111-124.

$[15]^{*}$ C. Nass, B. Fogg, and Y. Moon, "Can computers be teammates?", International Journal of Human-Computer Studies, 45(6), 1996, pp. 669-678.

[16]* J.C. Walliser, P. Mead, and T. Shaw, "The perception of teamwork with an autonomous agent enhances affect and performance outcomes", Proceedings of the Human Factors and Ergonomics Society Annual Meeting, 2017.

[17]* A. Panganiban, G. Matthews, and M. Long, "Transparency in autonomous teammates: Intention to support as teaming information", Journal of Cognitive Engineering and Decision Making, 14(2), 2020, pp. 174-190.

[18]* D. Siemon, R. Li, and S. Robra-Bissantz, "Towards a model of team roles in human-machine collaboration", Proceedings of the $41^{\text {st }}$ International Conference on Information Systems, 2020.

[19] S. Raisch, and S. Krakowski, "Artificial intelligence and management: The automation-augmentation paradox", Academy of Management Review, 46(1), 2021, pp. 192-210. [20] W. Frick, "When your boss wears metal pants", https://hbr.org/2015/06/when-your-boss-wears-metal-pants, accessed 03.05.2021.

[21] A. Baird, and L.M. Maruping, "The next generation of research on IS use: A theoretical framework of delegation to and from agentic IS artifacts", MIS Quarterly, 45(1), 2021.

[22] T. O’Neill, N. McNeese, A. Barron, and B. Schelble, "Human-autonomy teaming: A review and analysis of the empirical literature", Human Factors, forthcoming, 2020. 
[23]* A. Bogg, S. Birrell, M.A. Bromfield, and A.M. Parkes, "Can we talk? How a talking agent can improve human autonomy team performance", Theoretical Issues in Ergonomics Science, 22(1), 2020, pp. 488-509.

[24]* M. Demir, A.D. Likens, N.J. Cooke, P.G. Amazeen, and N.J. McNeese, "Team coordination and effectiveness in human-autonomy teaming", IEEE Transactions on HumanMachine Systems, 49(2), 2018, pp. 150-159.

[25] J.M. Leimeister, Collaboration Engineering: IT-gestützte Zusammenarbeitsprozesse systematisch entwickeln und durchführen, Springer, 2014.

[26]* M. Demir, N.J. McNeese, and N.J. Cooke, "The impact of perceived autonomous agents on dynamic team behaviors", IEEE Transactions on Emerging Topics in Computational Intelligence, 2(4), 2018, pp. 258-267.

[27] M. Poser, and E.A. Bittner, "Hybrid teamwork: Consideration of teamwork concepts to reach naturalistic interaction between humans and conversational agents", Proceedings of the $15^{\text {th }}$ International Conference on Wirtschaftsinformatik, 2020.

[28]* M. Dolata, M. Kilic, and G. Schwabe, "When a computer speaks institutional talk: Exploring challenges and potentials of virtual assistants in face-to-face advisory services", Proceedings of the $52^{\text {nd }}$ Hawaii International Conference on System Sciences, 2019.

[29] E. Salas, D.E. Sims, and C.S. Burke, "Is there a "big five" in teamwork?", Small Group Research, 36(5), 2005, pp. 555599.

[30]* S.K. Ötting, and G.W. Maier, "The importance of procedural justice in human-machine interactions: Intelligent systems as new decision agents in organizations", Computers in Human Behavior, 89, 2018, pp. 27-39.

[31]* A. van Wissen, Y. Gal, B. Kamphorst, and M. Dignum, "Human-agent teamwork in dynamic environments", Computers in Human Behavior, 28(1), 2012, pp. 23-33.

$[32]^{*}$ B. Wahn, and A. Kingstone, "Humans share task load with a computer partner if (they believe that) it acts humanlike", Acta Psychologica, 212, 2021, pp. 1-6.

[33]* T. Merritt, and K. McGee, "Protecting artificial teammates: More seems like less", Proceedings of the $30^{\text {th }} \mathrm{CHI}$ Conference on Human Factors in Computing Systems, 2012. [34]* M. Demir, and N.J. Cooke, "Human teaming changes driven by expectations of a synthetic teammate", Proceedings of the Human Factors and Ergonomics Society Annual Meeting, 2014.

[35]* C. Oh, J. Song, J. Choi, S. Kim, S. Lee, and B. Suh, "I lead, you help but only with enough details: Understanding user experience of co-creation with artificial intelligence", Proceedings of the $36^{\text {th }} \mathrm{CHI}$ Conference on Human Factors in Computing Systems, 2018.

[36]* D. Dellermann, A. Calma, N. Lipusch, T. Weber, S. Weigel, and P. Ebel, "The future of human-AI collaboration: A taxonomy of design knowledge for hybrid intelligence systems", Proceedings of the 52 $2^{\text {nd }}$ Hawaii International Conference on System Sciences, 2019.

[37] C. Nass, and Y. Moon, "Machines and mindlessness: Social responses to computers", Journal of Social Issues, 56(1), 2000, pp. 81-103.

[38] R. Wageman, H. Gardner, and M. Mortensen, "The changing ecology of teams: New directions for teams research", Journal of Organizational Behavior, 33(3), 2012.
[39] S.W. Kozlowski, and D.R. Ilgen, "Enhancing the effectiveness of work groups and teams", Psychological Science in the Public Interest, 7(3), 2006, pp. 77-124.

[40] E. Salas, T.L. Dickinson, S.A. Converse, and S.I. Tannenbaum, "Toward an understanding of team performance and training", in R.W. Swezey and E. Salas, eds.: Teams: Their training and performance, Ablex, 1992.

[41] J.R. Hackman, and J. Lorsch, "The design of work teams", in J. Lorsch, ed.: Handbook of Organizational Behavior, Prentice-Hall, 1987, pp. 315-342.

[42] C.P. Alderfer, "Organization development", Annual Review of Psychology, 28(1), 1977, pp. 197-223.

[43] J. Forster, Teams und Teamarbeit in der Unternehmung, Haupt, 1978.

[44] S.G. Cohen, and D.E. Bailey, "What makes teams work: Group effectiveness research from the shop floor to the executive suite", Journal of Management, 23(3), 1997, pp. 239-290.

[45]* G. Musick, T. O’Neill, B. Schelble, N. McNeese, and J. Henke, "Human-autonomy teaming: What happens when humans believe their teammate is an AI?", Computers in Human Behavior, 122, 2021.

[46] G. Pare, M. Tate, D. Johnstone, and S. Kitsiou, "Contextualizing the twin concepts of systematicity and transparency in Information Systems literature reviews", European Journal of Information Systems, 25(6), 2016, pp. 493-508.

[47] F. Rowe, "What literature review is not: Diversity, boundaries and recommendations", European Journal of Information Systems, 23(3), 2014, pp. 241-255.

[48] J. Webster, and R.T. Watson, "Analyzing the past to prepare for the future: Writing a literature review", MIS Quarterly, 2002, pp. xiii-xxiii.

[49]* E. Bittner, S. Oeste-Reiß, and J.M. Leimeister, "Where is the bot in our team? Toward a taxonomy of design option combinations for conversational agents in collaborative work", Proceedings of the 52 ${ }^{\text {nd }}$ Hawaii International Conference on System Sciences, 2019.

[50]* K.E. Schaefer, E.R. Straub, J.Y. Chen, J. Putney, and A.W. Evans III, "Communicating intent to develop shared situation awareness and engender trust in human-agent teams", Cognitive Systems Research, 46, 2017, pp. 26-39.

[51]* D. Derrick, and J. Elson, "Exploring automated leadership and agent interaction modalities", Proceedings of the $52^{\text {nd }}$ Hawaii International Conference on System Sciences, 2019.

[52]* M. Demir, N.J. McNeese, and N.J. Cooke, "Team situation awareness within the context of human-autonomy teaming", Cognitive Systems Research, 46, 2017, pp. 3-12.

[53]* E.K. Chiou, and J.D. Lee, "Cooperation in human-agent systems to support resilience: A microworld experiment", Human Factors, 58, 2016, pp. 846-863.

[54]* E.K. Chiou, J.D. Lee, and T. Su, "Negotiated and reciprocal exchange structures in human-agent cooperation", Computers in Human Behavior, 90, 2019, pp. 288-297.

[55]* D.A. Döppner, P. Derckx, and D. Schoder, "Symbiotic co-evolution in collaborative human-machine decision making: Exploration of a multi-year design science research project in the air cargo industry", Proceedings of the $52^{\text {nd }}$ Hawaii International Conference on System Sciences, 2019.

Articles included in literature sample are marked with (*) 